\title{
Misdiagnosed malignancy in transplanted organs
}

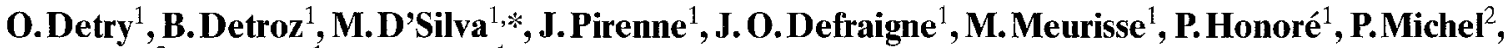 \\ J. Boniver ${ }^{2}$, R. Limet ${ }^{1}$, N.Jacquet ${ }^{1}$ \\ ${ }^{1}$ Department of Surgery and ${ }^{2}$ Department of Pathology, Centre Hôspitalier Universitaire, University of Liège, \\ Sart-Tilman, B-4000 Liège, Belgium
}

Received October 30, 1991/Received after revision May 8, 1992/Accepted June 25, 1992

\begin{abstract}
The case reports of three patients who received cancer-bearing organs at this institution are presented. A fourth recipient, who was to be transplanted with a cancerous kidney, was spared this disastrous complication. The relevant data regarding the donors is also alluded to, with special reference to the type and site of the primary malignancy. Following these case reports, the implications of these issues, their possible prevention, and further management are discussed.
\end{abstract}

Key words: Malignancy, donor - Organs, donors, malignancy - Donors, malignancy

Organ transplantation, a "miraculous" surgical procedure that occupies an increasingly important niche in the future of surgery, comes with a nasty package of unwelcome problems. The resolution of the technical aspects of the procedure, coupled with the increasing number of indications for organ transplantation, has led to a surge in the number of newer complications in the field. At present we not only have a perennial donor organ shortage but also a myriad of complications related to immunosuppression. One of the rare complications that must be addressed is the transferral of malignancy by grafting cancerous organs into recipients. The aim of this report is to highlight the issues related to the detection of the malignancy during organ procurement and other events that are intimately related to the donor operation. In addition, the variability in the management of these recipients post-transplantation is revealed. We hope this report will help to revive an interest in the search for such "malignant" donors. This "gray area" in transplantation needs greater exposure, for its presence shall be felt for years to come.

\footnotetext{
* Present address and address for correspondence: M. D'Silva, The Liver Unit, Queen Elizabeth Hospital, Edgaston, Birmingham B15 2TH, UK
}

\section{Case reports}

\section{Donor 1}

Donor 1 was a 30 -year-old female who succumbed to cerebral hemorrhage of nontraumatic origin. The salient and relevant past medical history included: (a) hyperandrogenism under treatment with corticosteroids, (b) the appearance of migraine headaches 1 year before death, and (c) the presence of intermittent hemoptysis 6 weeks before she presented as an organ donor. Her serum electrolytes were within normal limits and her renal and hepatic function profiles were normal too. A selective right carotid angiographic series disclosed a lesion occupying an avascular space in the right frontal region, supratentorially. A second finding of interest was the presence of premature basilar vein filling in the absence of grossly identifiable vascular malformation.

After obtaining consent for the multiorgan harvest, the liver and left kidney were explanted following standard organ procurement principles and techniques. The right kidney was rejected, owing to the presence of major congenital vascular and urological abnormalities. No other obvious intra-abdominal pathology was encountered at the time of organ procurement. Necropsy, which was performed the following day, revealed a nodule, $3 \mathrm{~cm}$ in diameter, in the rejected right kidney and three other hemorrhagic nodules located in the right lung. Their histopathological features were consistent with those of malignant trophoblastic tumor (choriocarcinoma).

Death was attributed to intracerebral hemorrhage from cerebral metastases of a misdiagnosed choriocarcinoma. Serum analyses conducted later revealed very high levels of beta-human chorionic gonadotrophin (BHCG) equalling $78000 \mathrm{IU} / \mathrm{I}$.

The liver and left kidney procured from donor 1 were transplanted into two different individuals - recipients 1 and 2 - at our center.

\section{Recipient 1}

This recipient, a 20 -year-old female afflicted with terminal nephropathy secondary to bilateral vesicoureteral reflux, was transplanted with the left kidney obtained from donor 1. The graft appeared healthy on inspection and palpation and did not reveal any gross pathology that might have disqualified it from the donor organ pool. After the results of necropsy were known, a computerized scan of the transplanted kidney was performed. This revealed a $2-\mathrm{cm}$ nodule in the organ possessing the features of a choriocarcinoma metastasis. 
Given this knowledge, immunosuppression was immediately terminated and the patient underwent a transplantectomy on postoperative day 12 . The explanted graft showed multiple foci of choriocarcinomatous metastases. The patient received nine injections of $3 \times 10.6 \mathrm{IU}$ interferon in an attempt to stimulate her immunity. Her serum BHCG level rose to $4000 \mathrm{IU} / \mathrm{l}$ by post-transplant day 16 with no detectable evidence of disseminated disease and actinomycin $\mathrm{D}$ was begun at a dosage of $1.25 \mathrm{mg} / \mathrm{m}^{2}$. Following an encouraging response after the initial 14 injections, tumoral resistance became manifest and the BHCG serum values rose again. By this time the patient had already received 14 injections of actinomycin D. It was then decided to initiate a combination chemotherapy regimen consisting of actinomycin D $\left(1.5 \mathrm{mg} / \mathrm{m}^{2}\right)$ and etoposide $\left(100 \mathrm{mg} / \mathrm{m}^{2}\right)$. With this regimen, $\mathrm{BHCG}$ levels fell dramatically and they have remained normal for the past 2 years. The patient was retransplanted at that point in time and since then shows no evidence of malignant disease.

\section{Recipient 2}

A 57-year-old male, who presented with hepatocellular failure secondary to alcoholic and viral cirrhosis, was transplanted with the liver from donor 1 . The donor liver, pretransplantation, appeared macroscopically normal and was transplanted without immediate untoward complications.

When the autopsy findings became known, ultrasound and tomography studies on the transplanted liver were conducted, but these did not disclose any metastasis. The patient's condition prohibited a retransplantation. However, the BHCG level rose from $580 \mathrm{IU} / 1$ on day 20 post-transplantation to $4300 \mathrm{IU} / \mathrm{l}$ on day 29 . At this point the patient received two separate injections of IV methotrexate (30 $\mathrm{mg} /$ day) spaced 3 days apart. Despite this, his BHCG jumped to $13000 \mathrm{IU} / \mathrm{l}$ on day 33 and then suddenly fell to $3000 \mathrm{IU} / \mathrm{l}$ on day 39 , when he died. The cause of death was attributed to overwhelming pulmonary complications. A formal autopsy revealed three hemorrhagic nodules in the right lobe of the liver and multiple pulmonary emboli. The postmortem diagnosis of the liver nodules was consistent with metastatic choriocarcinoma.

\section{Donor 2}

Donor 2, a 35-year-old female, presented for multiorgan harvesting at a different center in the country after being pronouced brain-dead following an abrupt, nontraumatic, cerebral hemorrhagic episode. Further interrogation disclosed that the patient had been treated with streptokin ase and heparin following the development of thrombophlebitis secondary to acupuncture. During the procurement, para-aortic adenopathy was noticed and this promptly underwent histopathological examination. In the meantime, the liver, both kidneys, the heart, and the pancreas were harvested. Bone was harvested as well.

The liver, heart, and kidneys were transplanted in four different centers. The results of the histopathological examination conducted on the para-aortic, pulmonary hilar nodes were positive for a disseminated, epidermoid epithelioma, invasive in nature, originating in the cervix uteri. Examination of the brain did not reveal metastatic lesions.

\section{Recipient 1}

The liver procured from donor 2 was transplanted at our center into a 25-year-old Asian male who presented with terminal acute hepatitis of probably both toxic and viral etiology. When the necropsy results became known, he was reinstated on the transplantation waiting list as an emergency. Computerized tomography and ultrasound studies were inconclusive and the patient was retransplanted 7 days later. No evidence suggestive of a malignant process was detected on histopathological examination of the liver. This recipient received triple immunosuppression, but no chemotherapy, following retransplantation. To date, a year later, the patient remains asymptomatic with no hint of malignant disease.

\section{Recipients for the heart and kidneys}

All data on organ donation were gathered by means of telephone conversations with the transplant coordinators at the centers where these organs were transplanted. One kidney was transplanted into a female patient at the donor center. This kidney was explanted on the 3 rd post-transplant day and the patient was retransplanted immediately. She died of a cerebral hemorrhage 20 days following the second retransplantation. Microscopic examination of the first kidney transplant proved negative for metastasis. The same result was encountered for the second kidney retransplant. Histopathological examination of the brain did not disclose any metastasis either. The second kidney was transplanted at another center. This graft was explanted within a week, the patient put on hemodialysis, and subsequently retransplanted after 14 months following the first transplant. The explanted graft was subjected to routine microscopic examinations that were inconclusive for malignancy. The heart, which was also transplanted at the fourth center, was left in situ and the recipient was followed rigorously. The patient remains free of malignant process to this day.

In analysis, none of the donor organs that were transplanted into the three recipients and subsequently explanted proved positive for cancer. In light of these findings, we could safely assume the same for the heart.

\section{Donor 3}

Donor 3 was a 55-year-old female who was pronounced brain-dead shortly after presenting to the emergency room at a hospital in another country with massive subarachnoidal hemmorrhage. The heart and both kidneys were procured from this donor. Save for four cysts detected at the time of harvest on one kidney, which proved negative for neoplasia on histopathological examination, no other gross or palpable intra-abdominal pathology was noticed by the donor team at organ procurement. One kidney was retained by the donor center while the second kidney and the heart were dispatched to two different centers. The kidney scheduled for transplantation at our center was scrutinized in the light of findings made known to us by the donor center. During the donor kidney examination, afifth additional lesion (measuring approximately $4 \mathrm{~cm}$ in diameter) was noted beneath one of the cysts. This lesion represented a solitary, yellowish nodule with no evidence of any discontinuity or disruption of its surface, suggesting that it had escaped the biopsy process. A frozen section confirmed the presence of a well-differentiated renal adenocarcinoma located in the middle portion of the kidney, the malignant process restricted essentially to the cortex but with a microinvasiveness in the renal parenchyma surrounding the lesion. Adjacent to the cancer lesion lay a simple serous cyst, previously detected and biopsied by the donor procurement team. Upon receipt of this information, further attempts to transplant the kidney were aborted, and the other recipient institutions were duly notified of our findings.

Fortunately, the kidney transplantation was also aborted at the donor center. An identical sequence of events followed at the heart recipient center. No formal autopsy was conducted on the donor as permission was withheld. Histopathological data were also unavailable for the second kidney and the heart.

\section{Discussion}

Transplantation has become the treatment of choice for a rapidly growing number of terminally ill patients, and forecasts predict an annual increase of $25 \%$ worldwide. This data translates into an increasing demand for donor 
organs and an increasing number of problems related to immunosuppression, among them the appearance of de novo cancers and, much more importantly, the inadvertent transplantation of organs bearing cancerous lesions into recipients. While the problem of de novo tumors is one that can be anticipated well in advance and one that can be explained to the recipient, the issue of transplanting cancerous organs is quite different in that it essentially results from an oversight and poses psychosocial issues to the recipient. In litigious societies such sensitive issues often wind up being decided in court. Although this problem is of far lesser magnitude than other transplantationrelated problems, it is nonetheless a very important issue that merits both attention and serious consideration. Some of the first case reports date back to the early pioneering years of transplantation when this avenue of transmission was not fully appreciated, but current case reports attest to its prevalence since that time $[2,3,8,10$, $13,15]$. Wilson and Penn [21] computed a $32 \%$ risk of transmitting a cancer with a transplanted allograft. Ten years later, this risk jumped to $43 \%$ [16]. By 1991, it was recalculated as $47 \%$ [18]. The significance of this problem is reflected in these figures. Despite the formulation of specific donor selection guidelines [5], their subsequent refinements $[11,12]$, and the variability in both their acceptance and application, tumor transferal continues to make its presence known in this advanced age of organ procurement and transplantation. Obviously, advances must be made in the identification of organ donors harboring a transplantable malignancy. Falling short of this goal, consensus must be reached as to the subsequent modalities of treatment for recipients bearing such grafts.

The areas that demand the most attention are the events surrounding the multiorgan donor procurement and the subsequent management of the recipient known to harbor a malignant transplant. A study of the relevant literature reveals a general trend that is predictable in relation to the circumstances surrounding the organ procurement procedure. The conventional battery of tests that are normally given in the work-up of the donor, i.e., chest X-ray, ECG, liver and renal function tests, are generally aimed at establishing donor suitability for procurement and donor monitoring during procurement. The results of these tests usually provide little, if any, evidence that might lead one to suspect there is an ongoing malignant process. It is not uncommon to note no gross intraabdominal pathology consistent with cancer that might alert the organ procurement team; oftentimes, the gross morphological features of the harvested organs are normal. The procurement procedure is seldom followed by a complete autopsy, for fiscal reasons. Thus far, published case reports are all one has to go by in terms of creating a cancerous donor profile.

Kidneys show an $8 \%$ incidence in macroscopic metastases originating in primary lesions localized to the breast, lungs, and gastrointestinal tract [20]. Barnes and Fox have rightly pointed out the underrating of the level of the actual problem since this figure does not take into consideration the microscopic metastases that often parasitize the donor organ [4]. The Cincinnati Transplant Tumor Registry (CTTR) data base comprises 135 reci- pients of transplanted organs bearing malignant lesions [18]. The reproducibility of the donor tumor in the grafted organs reached a level of $47 \%$, and the cadaveric organ pool was more likely to be implicated in the transmittal of malignancy. When considering the invasiveness of the transplanted malignancy, the highest proportion (55\%) belonged to the distant metastases group while the second largest group (37\%) included those in whom the neoplastic process was confined exclusively to the graft. The remaining $8 \%$ had, in addition, the involvement of adjacent structures. Penn goes on to indicate that while most recipients with documented widespread dissemination of the cancer died, $20 \%$ were saved. The origin of the primary cancers (in order of increasing frequency) were: kidney, lung, and colorectum. Other important sites included skin, breast, and ovary. The foregoing data are reason enough to consider a preventive approach to the donor operation by correctly establishing whether the donor is suitable.

Our well-meaning efforts to exclude cancerous donors are, at this time, most primitive, since our resources and priorities lead us to place emphasis on the hemodynamic stability and harvestibility of the potential donor. Retrospectively, simple observation is most often the only tool used that alerts the organ procurement team to the possibility of a cancer lesion $[1,7]$. Often, no intra-abdominal pathology is noted $[2,4,7,10,13]$. Autopsy, if done, is often conducted after the cancerous organs have been transplanted $[1,15]$. Generally, autopsy is never performed $[4,10,13]$ or is refused [3], and the cancer-bearing graft is stumbled upon accidentally $[8,13]$.

The donors we encountered in this report are unique in that both were young females in their reproductive years who presented with intracranial hemorrhage as the antecedent cause of brain death. While one was afflicted with a choriocarcinoma of the ovary, the other had carcinoma of the cervix uteri. At the moment of brain death, each had distant metastases. Gross observations of lymphadenopathy helped lead to the discovery of a malignancy in the second donor only. Necropsy in the first donor helped identify a malignancy never suspected during procurement. The lessons learned from this experience document the vital importance of a thorough medical history, followed by a complete physical examination of the donor. In the absence of a medical history (which is most often the case), a meticulous physical examination, coupled with current diagnostic aids, i.e., intraoperative ultrasound, must be relied upon. Had ultrasound been used in the first and second donors, this dreadful complication of transplantation might have been averted. In this respect we are in accordance with McCanty and colleagues [13]. We support the contention that all female donors in their childbearing years must be screened, specifically for BHCG levels and the presence of an occult asymptomatic cancer of gynecological origin. We also feel that every effort should be made to conduct an autopsy following the organ procurement, as we have shown its value in the two donors in this report. Baird et al. [1], Fox [7], Marsh et al. [10], and Barnes and Fox [4] have amply elucidated this issue time and again.

With special reference to the use of donors bearing primary intracranial cancers, we agree with Penn [18] that 
they could be considered for organ donation if, and only if, the tumor is primary with an absence of tentorial violation. Examples of systemic metastasis of central nervous system lesions are reported in the literature [9]. Our report does not encompass these pathologies. Finally, we feel that the use of donors with previously successfully treated cancer should be totally excluded from the donor organ pool, especially when their malignancies originate in the colorectum, since they have a high metastasizing capability and dormancy. It might help to recall that up to $30 \%$ of all patients with early stage colorectal cancer harbor hepatic micrometastasis [6]. As long as our diagnostic armamentarium remains inefficient in identifying occult dormant micrometastases and early recurrence, we must exclude this special potential organ donor. Transplanting grafts with these occult lesions into immunosuppressed hosts greatly enhances the chances of tumor dissemination, jeopardizing host survival. Malignant cells very rarely survive post-transplantation into healthy subjects. The normal immune system recognizes them as allografts and rejects the transplanted tumor. These facts were demonstrated by Martin et al. [11], Wilson et al. [22], and Matter et al. [12], who observed clinical, radiological, and histological regression of transplanted tumors by terminating their patients' immunosuppression.

The chronology of events surrounding donor 3 and the discovery of the cancerous lesions in the kidney allocated to our center attest to the fact that we are besieged by pressing problems of a logistical nature. Once again, owing to an increased level of suspicion of cancer int the donor organ, a dreadful complication was averted. The policy of "double-check" served well in our case and, in essence, serves as an example of the highest level of "preventive medicine" as applied to the field of solid organ transplantation. For the effective application of health care resources in the domain of prevention and for the future allocation of our health care dollars, one should bear in the mind the case report of donor 3 . In the absence of autopsy data on this donor, it would be impossible to ascertain whether the source of the nontraumatic subarachnoid hemorrhage was due to metastases. The circumstances surrounding the absence of autopsies are important, as there is no uniform law regulating this for the various countries that are part of the Eurotransplant network. In countries where presumed consent does not apply, obtaining permission for organ procurement is difficult enough; a subsequent attempt to obtain consent for a formal autopsy afterwards remains a formidable endeavor. These factors all contribute to the underestimation of this problem and to the incompleteness and inexactness of our existing data base.

Careful preselection of donors does not preclude the transferal of malignancy through organ transplantation. Considering the problem of a transplanted but previously unsuspected malignancy, several situations can be encountered that demand individualized management. Kidney allografts are generally explanted and immunosuppressive treatment terminated since there is a $46 \%$ chance that they contain tumors cells [17]. It is common to encounter reports where such a line of action is sufficient since immunological rejection of residual tumor cells can occur $[12,16,22]$; however, this is not always the case [19]. In the latter situation, chemotherapy has a definite role $[3$, 21].

Mocelin and Brandino's patient, transplanted with a living related kidney, died 2 months later with disseminated metastasis after post-transplantectomy coupled with immunosuppression removal and local irradiation [14]. Obviously, the time interval between transplantation and discovery of the malignant process is of utmost importance. Fortunately for most of the recipients discussed in this report, the discovery time was within 2 weeks. The kidney recipient's post-transplant course, with special reference to the elevated BHCG levels, is a case in point where transplantectomy and the discontinuation of immunosuppression were insufficient. Despite attempts to stimulate her immunity and a course of single drug chemotherapy, she still maintained high levels of BGCG overall. Only after instituting a double drug regimen including chemotherapy did she respond adequately.

The liver recipient is a good example of a case in which serum BHCG levels signaled residual metastatic activity. (Graft ultrasound and tomography studies did not reveal these metastases in situ.) In a situation like that of the recipient who received a liver from the donor with a cervical cancer, it is obviously difficult to monitor the graft for metastatic disease. In this particular case, the graft was free of metastatic lesions and chemotherapy was not used. Constant vigilance is mandated, however, since cancers can remain dormant for up to 3 yẹars [12].

With reference to the kidney grafts, we believe that specific chemotherapy must be initiated if the primary tumor is proven sensitive to treatment. Serum levels of a specific tumor marker can be very useful in the follow-up of widespread metastasis. Once the tumor undergoes remission, subsequent renal transplantation should be delayed for a 1-year, cancer-free interval, as Penn points out [17]. Consideration must also be given to pre-existing risk factors in recipients, such as their state of immunosuppression, owing to chronic uremia and blood transfusions, and the numerical order of the transplant.

Hepatic and cardiac allografts are unique in that they are not immediately expendable, and because of the lack of bridge-to-graft support to replace these functions, they must remain in situ until they can be explanted.

In the meantime, the termination of immunosuppression, the addition of chemotherapy, and tumor cell surveillance are the treatment options available. The conversion of a cancer to an allograft and the balance between graft rejection and tumor cell destruction is very variable and dubious at the very least. Long-term survival of widespread malignancy transferred by the liver is not common $[2,10]$. In the reported cases of heart allotransplants from donors with misdiagnosed cancer, no specific treatment was initiated. Immunosuppression was maintained and the recipients were closely monitored for metastasis, with none being found in the follow-up period $[3,10]$.

However, there have been exceptions [22], and this must be considered. It is interesting to note that the cardiac allograft from donor 2, transplanted at a different center, remains cancer-free, although the autopsy demonstrated bilateral pulmonary hilar lymphoadenopathy 
positive for metastasis from the "primary" lesion located in the uterine cervix. It is known that a cardiac allograft carries a low risk of metastatic spread and, as such, a conservative approach suits the situation well.

The three recipients transplanted at our institution provide compelling evidence that transplant centers both small and large need to be prepared for such an eventuality. Consensus on many of the issues raised in this report can only be reached when based on rigorous case detection and reporting endeavors.

In summarizing the events surrounding the donor operation, prevention is clearly the path to pursue to effectively utilize the available resources in the interest of the patient. These data explicitly expose the factors that contribute to the underestimation of this problem and to the incompleteness of our existing data base. In this fastchanging age of transplantation, where yesterday's relative contraindications are today's indications, where organ shortages make maximal utilization of available donor organs and minimal wastage imperative, it is conceivable that our well-intended efforts cloud our vision in the detection of cancer-bearing donor organs, placing a heavy burden on both the recipient and the health care system.

Acknowledgement. The authors acknowledge the assistance provided by Ms. Marie-France Hans.

\section{References}

1. Baird RN, White HJO, Tribe CR (1975) Renal carcinoma in a cadaver kidney graft donor. BMJ 2: 371

2. Baquero A, Foote J, Kottle S, Raja R, Mendez M, Noumoff J, Cavarocchi N, Cohn JB, Bannet AD (1988) Inadvertent transplantation of choriocarcinoma into four recipients. Transplant Proc 20: 98-100

3. Baquero A, Penn I, Bannet A, Werner DJ, Kim P (1988) Misdiagnosis of metastatic cerebral choriocarcinoma in female cadaver donors. Transplant Proc 20:776-777

4. Barnes AD, Fox M (1976) Transplantation of tumor with a kidney graft. BMJ 1: 1442-1444
5. Couch NP, Curran WJ, Hyg SM, Moore FD (1964) The use of cadaver tissues in transplantation. N Engl J Med 271: 691-695

6. Finlay IG, McArdle-S (1982) The identification of patients at high risk following curative resection for colorectal carcinoma. Br J Surg 69: 583

7. Fox M (1973) Renal carcinoma in a living kidney graft donor. Transplantation 15:523-525

8. Harvey L, Fox M (1981) Transferral of malignancy as a complication of organ transplantation: an insuperable problem? J Clin Pathol 34: 116-122

9. Jackson AM, Graham DI (1978) Remote metastases from intracranial tumors. J Clin Pathol 31: 794-802

10. Marsh JW, Esquivel CO, Makowka L, Todo S, Gordon RD, Tzakis A, Miller C, Morris M, Staschak S, Iwatsuki S, Mazariegos J, Kapadia S, Starzl TE (1987) Accidental transplantation of malignant tumor from a donor to multiple recipients. Transplantation 44: 446-450

11. Martin DC, Rubini M, Rosen VJ (1965) Cadaveric renal homotransplantation with inadvertent transplantation of carcinoma. JAMA 192: 752-754

12. Matter B, Zukoski CF, Killen DA, Ginn E (1970) Transplanted carcinoma in an immunosuppressed patient. Transplantation 9: $71-74$

13. McCanty TC, Jonsson J, Khawand N, Ali A, Edson M, Light J (1989) Transferral of a malignancy with a transplanted kidney. Transplantation 48: 877-879

14. Mocelin AJ, Brandino L (1975) Inadvertent transpiant of a malignancy. Transplantation 19:430

15. Oesterwitz HE, Lucius K (1991) Transmission of cancer with cadaveric donor kidneys. Transplant Proc 23: 2647

16. Penn I (1986) Transmission of cancer with donor organs. Transplant Proc 18: 471-472

17. Penn I (1988) Transmission of cancer with donor organs. Transplant Proc 20: 739-740

18. Penn I (1991) Cancer related to organ transplantation: summary and conclusions. In: Schmahl D, Penn I (eds) Cancer in organ transplant recipients. Springer, Berlin Heidelberg New York, p 179

19. Starzl TE (1969) Late results and complications. In: Starzl TE (ed) Experience in hepatic transplantation. Saunders, Philadelphia, p 369

20. Willis RA (1967) Pathology of tumors, 4th edn. Butterworth, London, $\mathrm{p} 175$

21. Wilson RE, Penn I (1975) Fate of tumors transplanted with a renal allograft. Transplant Proc 7: 327-331

22. Wilson RE, Hager EB, Hampers CL, Corson JM, Merril JP, Murray JE (1968) Immunologic rejection of human cancer transplanted with a renal allograft. N Engl J Med 278: $479-483$ 